\title{
Rhodes 1912 : Les mésaventures du Général d'Ameglio
}

http://revel.unice.fr/cmedi/document.html?id=155

Denis Bocquet

\section{Résumé}

La prise de Rhodes par l'armée italienne a lieu pendant la guerre italo-ottomane de 1911-1912. Cette conquête est la conséquence d'une stratégie de prise de position sur la côte d'Asie mineure. Cette île devait servir de monnaie d'échange avec la Sublime Porte contre un retrait de Tripolitaine et de Cyrénaïque et un arrêt du soutien à la résistance arabe de ces provinces.

Les premières administrations italiennes de Rhodes auront une tâche difficile face aux différents interlocuteurs : pouvoir de Rome, Empire ottoman, Etat grec, représentants des diverses communautés de l'île. Cette étude concerne la gestion du Général d'Ameglio.

\section{Abstract}

Capture of Rhode by the italian army takes place during the italo-ottoman war of 1911-1912. This conquest is the consequence of a setting up on the minor Asia's coast. This island should be used as change trade with the Sublime Porte against a withdrawal of Tripolitan and Cyrenaïc and a break-down to the arab opposition in these provinces.

The first italian governings of Rhode will have a difficult task over against interlocutors as : Rome, Ottoman Empire, greec State and représentatives of different island's communities. This study concerns général d'Ameglio's administration. 
La prise de Rhodes par l'armée italienne relève à la fois des hasards de la guerre, et d'une stratégie plus générale de prise de position sur la côte d'Asie mineure, en vue notamment d'un contrôle des ressources forestières. L'île devait officiellement servir de monnaie d'échange avec l'Empire ottoman contre un retrait total de Tripolitaine et de Cyrénaïque ainsi qu'un arrêt du soutien à la résistance arabe dans ces deux provinces. Cette ambiguïté fondatrice, ajoutée aux tensions de la société locale, rend la tâche des premiers administrateurs italiens de Rhodes extrêmement complexe.

La présente contribution s'attache ainsi à suivre les postures successives du général d'Ameglio, premier gouverneur de l'île, face à ses divers interlocuteurs : cabinet du Premier ministre à Rome, ministère italien des Affaires étrangères, Empire Ottoman, Etat grec, représentants de la communauté grecque de Rhodes, représentants des communautés musulmane et juive, ainsi que consuls français et britannique ou agitateurs nationalistes venus des quatre coins de la Méditerranée grecque. Les archives utilisées ici proviennent essentiellement de l'Archivio Centrale dello Stato, à Rome, et plus particulièrement de la Présidence du Conseil des Ministres ${ }^{1}$.

\section{Rhodes en 1912 : population, espace urbain et premiers choix de la tutelle italienne}

C'est dans le contexte de la guerre italo-ottomane de 1911-1912 que Rhodes est prise par l'armée italienne. L'île, tout comme ses voisines des Sporades méridionales (plus tard nommées Dodécanèse) ne semble pas constituer au départ un véritable objectif de guerre italien, même si, depuis de nombreuses années, diverses missions archéologiques et géographiques de scientifiques et espions avaient contribué à repérer le terrain et à préparer une éventuelle occupation. Rhodes, au début du XXe siècle, est une ville ottomane. D'après un rapport militaire, elle compte environ 13500 habitants en $1912^{2}$. Près de la moitié appartiennent à la communauté des chrétiens orthodoxes Grecs (6000).

La ville compte aussi environ 5000 musulmans, et 4000 juifs. Elle se distingue en cela des campagnes de l'île, et des autres îles de l'archipel, où est prédominant l'élément orthodoxe. A Rhodes-ville seulement, existe une réelle diversité communautaire. Pour le reste de l'île, on ne compte que deux villages musulmans (Kalamona et Isguru), qui n'abritent que respectivement 185 et 100 habitants. Rhodes est donc très différente, par sa physionomie et par son organisation sociale, du reste des îles occupées par l'Italie. Voici les données compilées par l'armée italienne au 21 septembre $1912^{3}$ :

Ville de Rhodes et faubourgs

Maisons orthodoxes 771 Présents 3605 Absents 641

Tot. 4246

Maisons israélites 596 Présents 3692 Absents 598

Tot. 4290

Maisons catholiques 56 Présents 269 Absents 49

Tot. 318

Maisons musulmanes 820 Présents 3805 Absents 1085

Tot. 4890

1. - Désormais A.C.S., P.C.M.

2. - Comando della 6a Divisione speciale, Cenni Monografici sulle Sporadi meridionali occupate dalle truppe italiane durante la guerra italo-turca, Rhodes, 1912, A.C.S., P.C.M., 1912, Tripolitania.

3. - Comando della 6a Divisione speciale. Contributo monografico per lo studio politico ed economico dell'isola di Rodi, 1913. A.C.S., P.C.M., 1913, fasc 1-2 . 
On a donc un total de 2243 maisons, 11371 présents, 2273 absents, soit 13744 habitants. Pour l'île, sauf ville de Rhodes, on a les chiffres suivants : 13000 orthodoxes et 1600 musulmans, ce qui porte la population totale à 28344 habitants. Les catholiques sont pour moitié Français (149). Les autres sont Arméniens Ottomans (60) et Italiens (53). La description italienne que l'on trouve dans le document de 1913, atteste la répartition de cette population en quartiers selon l'appartenance confessionnelle. On a ainsi des quartiers grecs, un quartier juif et un quartier musulman. La population grecque se répartit ainsi par paroisses :

Ste Anastasia et St Jean : 184 maisons, 400 hommes, 430 femmes, 181 absents, 1011 habitants

Neokori : 192 maisons, 434 hommes, 432 femmes, 169 absents, 1035 habitants

St Georges et St Nicolas : 160 maisons, 347 hommes, 359 femmes, 144 absents, 706 habitants

Métropolite : 235 maisons, 609 hommes, 594 femmes, 147 absents, 1350 habitants

Notons que la densité d'habitants par maison est beaucoup plus forte dans le quartier Métropolite. Petite ville, port secondaire du trafic de l'Est méditerranéen, le long de la côte d'Asie mineure, Rhodes présente de nombreuses caractéristiques qui la rattachent à d'autres villes de l'Empire ottoman : siège administratif d'un vilayet, gouvernée par un wali, elle est dotée des principales institutions urbaines, judiciaires et administratives du système ottoman.

La ville compte de nombreux fonctionnaires, de la douane à la régie des tabacs, du tribunal religieux et administratif à la garnison militaire. La répartition de la population par quartiers, selon l'identité confessionnelle, rapproche Rhodes d'autres villes ottomanes. Sous l'Empire ottoman, les îles étaient dotées de divers privilèges, institutionnels et fiscaux, accordés pour certains dès le XVIe siècle. On n'y payait pas un certain nombre de taxes, et y était exempté de service militaire. Une forme d'autonomie avait aussi été concédée. Les communautés insulaires avaient le droit d'élire leur démogérontie, ou conseil des Anciens. Ces privilèges régaliens avaient été accordés par les souverains ottomans comme dérogation à la règle générale de l'Empire.

Après les troubles de 1828, ces privilèges furent confirmés en 1834 par le sultan Mahmud. En 1836 les îles furent rassemblées dans le Vilayet de l'Archipel Ottoman ${ }^{4}$. Les tribunaux locaux étaient indépendants, conduits par des juges nommés par les Anciens. Le gouverneur de l'île de Rhodes avait peu de pouvoirs face aux communautés locales. Il était représenté dans chaque île par un mudir, qui validait les délibérations des Anciens, sans grand pouvoir d'après le rapport italien de 1913. En 1873, l'intégration au droit commun ottoman provoqua des troubles, mais des exemptions vinrent rapidement tempérer les réformes. En 1910, une commission financière ottomane décide de proroger de fait les privilèges.

La ville de Rhodes est en décalage par rapport au reste des îles, par la composition de sa société urbaine et les privilèges séculaires accordés, et sans cesse confirmés, qui ont apporté des dérogations, et des obstacles, à l'application de la loi municipale et provinciale ottomane de 1877.

En 1912, on a donc encore à Rhodes une Démogérontie, conseil des Anciens à base communautaire grecque, un conseil des Anciens musulmans, et un conseil communal (communautaire) israélite. A côté de ces institutions de gestion sociale et urbaine, on trouve une municipalité, dont les fonctions se confondent parfois de fait avec celles des notables communautaires. Les Italiens de 1912-1913 décrivent la vie à Rhodes comme marquée par une absence de communication entre communautés :

\footnotetext{
"Entre un groupe ethnique et l'autre, il n'existe pas de contact matériel ou moral d'aucune sorte. Chacun essaie de s'atteler à son activité dans le cercle limité de ses semblables, détestant se mélanger avec ceux qu'il juge de race et de catégorie inférieure. Chaque communauté a une tradition à préserver, un droit de suprématie à vanter. (...) Les îles que nous occupons encore représentent un échantillon du monde levantin, avec cette particularité que les conditions géographiques ont rendu chacun plus individualiste, et empêché l'agrégation démographique que connaissent les côtes de l'Asie mineure " 5.
}

4. - Comando della 6a Divisione speciale. Contributo monografico per lo studio politico ed economico dell'isola di Rodi, 1913 , p.74. A.C.S., P.C.M., 1913, fasc 1-2 . 
Le rapport souligne aussi l'imbrication, pour les Grecs, entre vie religieuse et vie civique : “ la religion et la politique se sont fondues en une unité indestructible, les aspirations patriotiques ont pris une forme mystique ". Le rapporteur voudrait que l'Eglise de Rhodes soit soustraite au patriarche de Constantinople. Les musulmans, en nombre supérieur aux Grecs dans la ville de Rhodes même, selon une des deux sources italiennes disponibles, semblent posséder leurs terrains en ville et à proximité immédiate.

Le rapport de 1913 affirme que deux tiers des bâtiments privés de la ville appartiennent à des musulmans. Ceci expliquerait aussi pourquoi les Turcs ont eu tant de succès aux élections municipales ottomanes, fondées sur un système censitaire, au cours des dernières années avant l'occupation italienne. Au cours des années ayant précédé 1912, des crétois musulmans se sont de plus réfugiés à Rhodes, et ont renforcé la communauté locale.

Le rapport dit :

\footnotetext{
“ les musulmans arrivés après la conquête ottomane, il y a plusieurs siècles, habitent tous en ville, où ils possèdent maisons et terrains. En général ils sont disciplinés, obéissent aux lois, et sont indifférents au système de gouvernement. Ils se consacrent à leurs affaires, et habitent un quartier parfaitement séparé, sauf ceux qui possèdent des villas hors de la ville, seule manière pour Grecs et Turcs de se mélanger" "6 . Le jugement sur les crétois exilés est plus sévère. Ils n'ont pas accès à la notabilité. Les crétois sont intelligents, actifs, mais fanatiques" $"$.
}

Le même rapport de 1913 décrit également la situation des juifs de la ville. Originaire d'Espagne, arrivée dans l'île au XVIe siècle, la communauté occupe un quartier distinct :

\footnotetext{
“Elle compte parmi elle les principaux négociants et banquiers de l'île, qui contrôlent presque tous les échanges avec l'Europe, et entre l'île et la côte anatolienne. Elle est obéissante aux lois et à l'Autorité, et partisane d'un gouvernement qui ne l'opprime ni matériellement, ni moralement, lui consentant un juste développement de ses aspirations religieuses, économiques et éducatives. (...)
}

\begin{abstract}
La communauté, depuis l'occupation italienne, a donné des signes manifestes d'une profonde satisfaction pour l'équanimité avec laquelle elle a été traitée. Un gouvernement animé d'un esprit religieux excessif contraindrait les israélites à émigrer sur les côtes de l'Asie mineure, ce qui serait un dommage considérable pour l'activité économique de l'île, qui tire les plus grands bénéfices de l'activité intelligente de la communauté juive",
\end{abstract}

Le rapport de 1913, issu de l'administration militaire, est également intéressant dans la vision qu'ont les Italiens de l'organisation administrative :

\begin{abstract}
“ A la suite du Traité de Berlin, l'Angleterre a occupé Chypre, et a adopté, pour ce qui concerne l'organisation administrative et politique, une attitude adaptée à la connaissance du milieu, se rendant compte que toute introduction directe du Gouvernement dans les affaires locales aurait été nocive. Elle a ainsi concédé une vaste autonomie aux institutions municipales, afin de ne pas offenser la susceptibilité d'un peuple par nature hypercritique, aimant, par, tradition antique, discuter, parlementer, et ne s'est réservée que le contrôle suprême, confié à un Haut commissaire, responsable seulement devant Londres ".
\end{abstract}

C'est ce type de solution que voudrait pour Rhodes le rapporteur, proche collaborateur du général d'Ameglio.

\title{
Le général d'Ameglio entre nationalismes, communautarismes et intérêts contradictoires
}

Les soucis de l'administration militaire italienne avec l'élément grec avaient commencé dès les premières semaines de leur présence dans les îles. Après les premiers moments de célébration nationaliste grecque, tolérés par des troupes qui, de toute façon, ne contrôlaient encore que les ports, la nécessité de prendre position face aux velléités de rattachement à l'Etat grec, issues 
principalement des petits villages de l'île, devient rapidement évidente.

Dès le mois de juin, des rapports font état au Premier ministre Giolitti des activités de divers agitateurs nationalistes grecs. Un rapport secret l'informe de l'action de certains responsables religieux orthodoxes visant à promouvoir un rattachement à la Grèce'. Il y est question du nouveau Métropolite grec de Rhodes, nommé par le Patriarche d'Istanbul. Le général d'Ameglio, se pose la question de savoir s'il faut autoriser le débarquement d'un personnage aussi farouchement nationaliste. Giolitti, conscient que refuser serait tomber dans un piège, et ne ferait qu'aggraver la situation, signe lui-même l'autorisation.

Un rapport secret du 3 juin avertit des risques d'une inflation nationaliste grecque : "A qui connaît l'influence des autorités religieuses dans leur diocèse, il ne paraîtra pas superflu de suivre avec attention leur conduite à Kalimnos et à Rhodes "10. Les déboires des autorités italiennes dans ce domaine, et du général d'Ameglio en premier chef, ne font que commencer. Pendant plusieurs années, les représentants de la hiérarchie orthodoxe dans les îles occupées conduisent une forte politique d'opposition à l'occupation italienne, en liaison avec les autorités orthodoxes à Istanbul, le consulat de Grèce, et la presse nationaliste athénienne.

Une autre difficulté pour les Italiens, dans leur rapport au nationalisme grec, vient rapidement des institutions communautaires des îles et villages dans lesquels l'élément grec domine. Plusieurs assemblées locales organisent des réunions extraordinaires pour à la fois célébrer la fin de la domination ottomane, et demander un pas supplémentaire en direction du rattachement à la Grèce.

Dans les premiers jours de juin 1912, aussi, la " Chambre des Députés de l'île de Samos ”, restée possession ottomane, fait parvenir aux " puissances protectrices de l'île " une pétition détaillée, dans laquelle il est demandé que la Russie, l'Angleterre et la France veillent au respect de l'autonomie samienne face à la présence de troupes ottomanes ${ }^{11}$. Bien qu'il n'y ait rien là contre les Italiens directement, on comprend que le gouvernement Giolitti s'en émeuve : si, dans le contexte de la guerre italo-ottomane, les communautés locales insulaires grecques en appellent à la protection des puissances rivales de l'Italie en Méditerranée, un mouvement très embarrassant risquerait de s'amorcer.

A Rome, on prend la menace d'une propagation de ce mouvement très au sérieux. L'Italie veut à tout prix éviter de donner un prétexte à ses rivales européennes pour intervenir dans sa portion du jeu méditerranéen. A partir de ce moment, latitude plus grande est donnée aux autorités religieuses et aux institutions locales d'exprimer leur sentiment national grec. Malgré qu'il en ait, devant gérer sur place ce qui de Rome paraît plus simple, le général d'Ameglio obtempère.

Cette stratégie semble d'ailleurs la seule possible. La présence à Rhodes de militants politiques samiens renforce les craintes des services du renseignement militaire d'une tentative d'implication des puissances tutélaires. La crainte la plus forte pour le gouvernement italien est bien, à ce moment-là, d'une implication française ou anglaise dans les affaires insulaires de la mer Egée. Dès 1912, le gouvernement italien doit trouver une position médiane entre trois forces antagonistes : l'Empire ottoman, avec qui elle signe un traité de paix à l'automne, les puissances européennes, qu'il ne peut se permettre d'affronter, et le nationalisme grec, puissant mouvement politique dont les représentants, aussi bien à Rhodes qu'à Athènes, Istanbul ou New-York, savent jouer sur tous les tableaux.

Autant en Tripolitaine, conquise lors de la même guerre, l'action italienne prend dès les premières semaines un tour éminemment colonial, autant à Rhodes, les autorités italiennes choisissent de jouer profil bas. Le rapport, dans la ville de Rhodes, aux diverses institutions du gouvernement urbain en découle. Au cours de la deuxième moitié du mois de juin 1912, l'agitation nationaliste grecque dans

9. - A.C.S., P.C.M., 1912, Tripolitania.

10. - Idem.

11. - Copia della protesta della Camera dei Deputati di Samos alle potenze protettrici dell'isola, A.C.S., P.C.M., 1912, Tripolitania. 
les îles occupées par l'Italie se développe d'une manière très organisée, reprenant les cheminements bureaucratiques des institutions de l'autonomie ottomane.

Le 21 juin, un télégramme confirme que le précédent samien constitue le modèle de la protestation Egéenne. C'est Giolitti qui en avertit d'Ameglio à Rhodes : il a reçu des services de renseignement italiens à Athènes la confirmation de la tenue d'une réunion, à Patmos, sur le modèle de celle de Samos :

\footnotetext{
“ Un télégramme d'Athènes m'informe que des représentants des douze îles que nous occupons ont tenu congrès à Patmos, et ont le projet de s'adresser à vous pour demander l'union à la Grèce, et si cela est impossible, l'autonomie, avec comme symbole le drapeau à croix blanche sur fond bleu. Tout en vous demandant de m'informer immédiatement quant à la véracité de ces informations, je vous demande d'user de la plus grande réserve quant à un sujet aussi délicat $" 12$.
}

Le message au gouverneur militaire d'Ameglio est aussi un reproche : Giolitti est troublé de n'avoir pas été informé par son représentant sur place de la tenue de telles réunions. Cette agitation trouve sa force dans l'activité d'institutions locales sises dans des villages et des îles à la population majoritairement grecque, où les institutions locales sont l'expression de la volonté de cette population. Rhodes étant à part, la difficulté pour les troupes italiennes n'en est que plus grande, et la dispersion des Sporades méridionales constitue un grand désavantage.

Après avoir reçu le télégramme de Giolitti, le lendemain, Ameglio fait semblant de prendre les devants, et de ne pas encore avoir lu ce que lui écrit le Président du conseil. Il envoie à son tour un télégramme signalant la réunion :

\footnotetext{
“Dans un monastère de Patmos, dix représentants autoproclamés des îles occupées se sont rencontrés pour rédiger une motion destinée à être présentée au gouvernement italien et aux puissances. Dans ce texte, après l'expression de leur reconnaissance à l'Italie, ils déclarent que l'aspiration nationale des habitants des îles est de s'unir à la Grèce. Ils proclament la complète autonomie, sur la base de promesses que j'aurais faites. Ils donnent le nom d'Etat de l'Egée aux îles, et adoptent le drapeau bleu à croix blanche. Ces représentants n'avaient aucun caractère officiel. Les maires n'ont pas participé à la réunion. Selon toute probabilité, elle a été organisée par le vice-consul grec Liatis, venu tout exprès dans les îles, à la suite de notre occupation. Les délégués, qui ont annoncé vouloir me présenter la motion, ne sont pas encore arrivés. Je ne les recevrai pas. Toutes les institutions de Rhodes ont été averties que je serai sévère si de telles manifestations devaient se reproduire. Elles ont émis une déclaration dans laquelle elles confirment que je n'ai jamais parlé d'autonomie. Le consul général de Grèce a promis de faire rappeler Liatis à Athènes avant que je ne le fasse arrêter. Parmi des participants à la réunion de Patmos, un est venu d'Alexandrie "13.
}

D'Ameglio tient désormais à prendre l'initiative. Surpris de voir que Giolitti avait d'autres canaux d'information efficaces, il a été pris en faute. C'est toute son attitude qui est potentiellement remise en question. Il fait montre, dans ce télégramme, d'une emphase presque ridicule, afin de tenter de s'attirer de nouveau les faveurs du Premier ministre. Les informations qu'il donne permettent aussi de préciser notre description de la situation.

On a donc, à l'été 1912, une agitation nationaliste grecque dont le cœur est constitué des îles les plus périphériques par rapport à la présence italienne, et qui se nourrit, de manière très organisée, très ottomane aussi, du fonctionnement des institutions locales communautaires, et d'un autre côté les services du général d'Ameglio qui contrôlent de près les institutions de Rhodes-ville.

L'agitation nationaliste est assurément encouragée par la Grèce. En retour, les Italiens, sur place, tentent de faire jouer en leur faveur d'autres institutions ottomanes : les municipalités et la représentation communautaire là où elle n'est pas grecque, ou alors suffisamment mise sous tutelle. Mais la position d'Ameglio est fragilisée à partir de l'été 1912, tant à Rome que sur place. Le général n'améliore pas son cas dans les semaines qui suivent, et la consultation des archives de Giolitti démontre qu'il devient un grand souci. A nourrir les réprobations, il y a d'abord une interview donnée par le général au magazine Rietch ${ }^{14}$. Le journaliste, un certain Witclof, lui attribue les propos suivants :

14. - Numéro du 22 juillet-4 août 1912. A.C.S., P.C.M., 1912, Tripolitania. 


\begin{abstract}
“Nous aimons le soleil, et nous sommes venus dans ce pays pour libérer ce peuple malheureux, qui n'a pas vu le soleil pendant tant d'années. Nous avons apporté ici la liberté, et la possibilité pour tous de vivre sous une loi unique. Nous avons apporté ici la plus haute justice, et nous espérons qu'elle triomphera. (...) Je ne sais pas comment finira la guerre. Mais après tout ce que nous avons fait ici, on peut affirmer avec assurance que Rhodes ne peut être rendue aux Turcs. Cela constituerait une injustice colossale envers la population locale " 15 .
\end{abstract}

Au ministère des Affaires étrangères, qui signale l'affaire à Giolitti le 20 août, on est furieux ${ }^{16}$. En pleine négociation de ce qui deviendra le Traité de Lausanne, le général qui représente l'Italie à Rhodes en nie les fondements ! Le ministère des affaires étrangères confirme que d'Ameglio a bel et bien tenu ces propos, et qu'il ne pourra pas de nouveau se cacher derrière d'habiles dénégations : "Le général ne peut, ni doit, s'exprimer dans ces termes, et encore moins formuler de telles opinions quant à la question de la restitution ou non des îles à la Turquie "17. A la suite des réprobations qui lui sont envoyées, d'Ameglio se tait pendant plus de trois mois, et se contente d'envoyer à Rome de très bureaucratiques télégrammes.

Après la signature du Traité de Lausanne, la question s'est pourtant de nouveau compliquée : d'Ameglio est cette fois accusé par l'opinion grecque de ne faire que maintenir l'ordre et expédier les affaires courantes dans l'attente d'un retour d'une garnison ottomane à qui seraient rendues les îles. A partir du mois de novembre, il semble que les responsables grecs s'emploient à tout faire pour éviter cette éventualité, et même à préparer une résistance populaire dans les campagnes. D'Ameglio signale le 27 octobre que le consul de Grèce à Rhodes, Papadaki, tente de mettre sur pied les infrastructures d'une possible résistance anti-ottomane.

$\mathrm{Au}$ ministère des Affaires étrangères, on considère la question avec sérieux, et commence à expliquer à Giolitti “ qu'il ne sera pas possible d'étouffer le sentiment de répugnance insulaire face à la perspective d'un retour des Turcs. De nombreux habitants émigrés, originaires des îles, se présentent dans nos bureaux en pleurant, nous suppliant de sauver leurs familles et de laisser la Grèce envoyer des troupes au moment du départ des Italiens " $"$.

A la fin de ce même mois de novembre 1912, de sérieux incidents se déroulent à Nisiros. Une manifestation, organisée par le maire, et autorisée par d'Ameglio à condition qu'aucun discours ne soit tenu, réunit plusieurs centaines de patriotes grecs ${ }^{19}$. En novembre 1912 toujours, les services de renseignement italiens interceptent une lettre de la Démogérontie de Rhodes à des correspondants politiques à Athènes, preuve de l'organisation d'une opposition forte contre les Italiens ${ }^{20}$. La réponse de Drakakis, évoquant les “verres amères offerts par une nation sœur”, confirme la consistance de ce réseau d'expression politique ${ }^{21}$.

Les espoirs cependant de voir le sort de Rhodes résolu en relation avec les négociations sur la Macédoine sont vains. Drakakis propose d'utiliser les institutions municipales comme chambre de résonance. Il suggère une démission en bloc de tous les maires, susceptible d'attirer l'attention " en Italie des libéraux et des socialistes ${ }^{~} 22$. Les paroisses, autres institutions de représentation

16. - R. Ministero degli Affari Esteri. Direzione Generale degli Affari Politici. Div. III, Sez. II, $n^{\circ}$ 58784, A.C.S., P.C.M., 1912, Tripolitania.

17. - Idem.

18. - A.C.S., P.C.M., 1912, Tripolitania, 1-11-1912.

19. - A.C.S., P.C.M., 1912, Tripolitania, Rapport Ameglio à Giolitti, 24-11-1912.

20. - Lettre à G. Drakakis et G. Paolides, A.C.S., P.C.M ., 1913, fasc. 1-2. Les huit démogérontes signataires sont : S. Pavlides, G.C. Papadopoulos, N. Giorgilas, S. Parasckevas, Ajacatzicas, M . Atanassiadis, Teodore Frarakis, D. Anastassiadis.

21. - Réponse de Drakakis au démogéronte rhodien Anastassiadis, 26 novembre 1912, A.C.S., P.C.M., 1913, fasc. 1-2.

22. - Idem. 
communautaire grecque, se mobilisent également.

Les paroissiens de Rhodes-ville envoient ainsi à d'Ameglio, en octobre 1912, une pétition demandant leur rattachement à la mère patrie ${ }^{23}$ :

\begin{abstract}
“ Plébiscite des paroissiens de la Métropole, Ste. Anastasia, St. Georges supérieur, S. Nicolas et St Georges inférieur. Les soussignés habitants des paroisses du chef-lieu St Anargiri de Rhodes, faisant encore preuve ici de leurs sentiments, en liaison avec la résolution de Patmos du 4 juin 1912 prise par les représentants légitimes des îles occupées par l'Italie, expriment : leur ardent vœu d'être unis à la mère Hellade. Si cela est impossible, pour des raisons internationales, le souhait que les gouvernements libéraux des grandes puissances, conformément au droit des Nationalités, concèdent une pleine autonomie politique, garantie par un traité international. Reconnaissants envers la bienfaisance de la sœur Italie, le vœux que celle-ci les soutienne dans la consolidation de leur autonomie politique. Leur mandat donné à la Démogérontie du chef lieu de Rhodes pour la transmission du présent plébiscite aux Grandes Puissances ”.
\end{abstract}

Le choix de l'Italie, à partir de ce moment, est d'ores est déjà celui de ne pas partir, tout en évitant les signes affichés d'une souveraineté sur l'île. La situation en Tripolitaine, avec la persistance d'une résistance arabe, dont il est facile de supposer qu'elle a l'appui de la Porte, donne tous les prétextes nécessaires. Les archives de l'E.U.R., pour ce mois de novembre 1912, recèlent d'une protestation, en grec, écrite par un habitant de Rhodes ${ }^{24}$ :

\footnotetext{
"Quand votre honorable terre s'est battue pour la possession de la province de Tripolitaine, en déclarant la guerre à la Turquie, elle a annoncé au monde que la guerre était pour le progrès et pour la civilisation. Vous ne deviez ni l'un ni l'autre à cette région. Les événements postérieurs ont démontré qu'à l'évidence Votre Nation, soyons juste, en même temps qu'elle donnait de la civilisation en recevait en échange les fruits. Quand en mai dernier, la guerre, à cause de vos choix, s'est transportée dans la mer Egée, et que la glorieuse armée italienne a occupé l'île de Rhodes, le valeureux et gentil condottiere Ameglio, vrai chrétien et vaillant soldat italien, voyant les terribles conditions dans lesquelles était réduite cette île, autrefois romaine, depuis longtemps réduite en esclavage, ressentant le devoir sacré envers Dieu et envers les hommes de sauver l'île abandonnée, assurait aux pauvres habitants sur son honneur, que jamais ces belles îles, tant qu'il serait là, ne serait " rendues au barbare dominateur". Mais Vous, oubliant les origines historiques de l'île de Rhodes, avez, dans un moment cruel d'amnésie, signé la condamnation de tant d'âmes qui avaient en vain placé leur espoir en vous. Il est ignominieux, pour le peuple italien de supporter que l'on négocie son honneur aux dépens d'habitants sans défense. (...) Alors que vous arrachez la Tripolitaine à la Turquie pour donner la civilisation aux Noirs, vous vous apprêtez à rendre nos îles ! (...) Il n'y a pour vous, déshonorés, qu'une seule voie : “Eloignez-vous tout de suite des îles, et donnez-les à l'honorable et glorieuse flotte grecque (...) ”.
}

La lettre est signée : un habitant trahi.

Cette lettre reflète bien la situation dans l'île. La position d'Ameglio devient intenable. Lui qui a failli payer de sa place sa réticence à rendre Rhodes aux Ottomans, qui a dû ensuite se rendre impopulaire en faisant mine de la rendre, doit désormais gérer une situation dans laquelle plus personne ne lui fait confiance : ni le gouvernement italien, ni l'opinion grecque, ni les éléments ottomans. D'Ameglio continue, de plus, d'être la cible de violentes attaques de la presse athénienne, qui l'accuse de mener une féroce répression contre les activités nationalistes dans les îles.

L'année 1913 est marquée par une intensification des protestations contre l'occupation italienne. Celles-ci proviennent désormais non seulement des îles mêmes et de Grèce, mais aussi de toutes les communautés grecques de Méditerranée, d'Istanbul à Alexandrie, ainsi que des nombreux émigrés rhodiens en Amérique. Au mois de janvier, le journal athénien Nea Imera se fait l'écho d'une démarche de l'assemblée des anciens de Rhodes, qui vote une délibération adressée au Ministre des Affaires étrangères grec ${ }^{25}$.

23. - A.C.S., P.C.M., 1913, fasc. 1-2.

24. - A.C.S., P.C.M., 1912, Tripolitania, lettre anonyme du 28-11-1912.

25. - A.C.S., P.C.M., 1913, Fasc. 1-2, Rodi. Rapport du Comando dei Carabinieri Reali dell'Egeo sur l'article de Nea Imera du 5 janvier 1913. 


\begin{abstract}
“Excellence, la démogérontie de Rhodes, représentant légal des habitants de l'île, réunie en ce 22 décembre 1912 (4 janvier 1913), ayant pris en considération le fait que, ces jours-ci, devra se jouer le sort des îles de la mer Egée entre les Grandes Puissances dans la capitale de la grande et libérale nation britannique, et manifestant le sentiment des habitants, rassemblant son courage, a l'honneur d'élever la voix et d'exprimer la seule et séculaire aspiration du peuple de l'hellénissime île, en faveur de son annexion à la Grèce mère, seule solution susceptible d'apporter à l'île paix durable et prospérité. Plaçant son espoir dans les sentiments libéraux des représentants des Grandes Puissances et implorant leur secours et leur protection, demande que conformément au droit des peuples soit écoutée cette juste et séculaire aspiration du peuple de Rhodes " 26.
\end{abstract}

La conférence de Londres ne change en fait rien à la question. Les puissances européennes renoncent à mettre l'Italie en difficulté et de fait confirment sa tutelle sur Rhodes. Ce qui est intéressant, c'est encore une fois de voir le mode d'expression de l'aspiration nationale grecque, au travers des institutions traditionnelles, à base communautaire, partiellement insérées dans le système municipal ottoman. Ce genre d'appel a aussi dans l'opinion grecque une forte résonance, et rend la tâche des Italiens toujours plus ambiguë, entre provisoire et définitif, protection supposée face aux Ottomans et répression des aspirations nationales.

Dans ce contexte, le Ministère des Affaires étrangères italien demande un rapport précis sur la situation $^{27}$. Pour la première fois sont évoquées les autres communautés. Pour l'auteur du rapport, l'opinion insulaire est plus divisée que ne le laisse voir l'activité nationaliste grecque. Il souligne que, si les intellectuels grecs sont favorables au rattachement, les populations locales sont, au contraire, favorables à une simple autonomie, au nom du refus du service militaire. "Turcs et Israélites préfèreraient la souveraineté italienne".

Pour la première fois, ce rapport télégraphique évoque aussi l'importance de Rhodes comme position privilégiée face à la côte anatolienne, en vue de la pénétration européenne : "Iles à la Grèce mettraient celle-ci en condition aider deux millions Grecs disséminés côtes occidentales Asie Mineure, dérangerait pénétration pacifique Allemagne France Angleterre. Permettrait Grèce augmenter forces navales altérant actuel équilibre naval Méditerranée "28. Ce rapport souligne aussi qu'une puissance comme la France mettra au premier plan de ses intérêts la lutte contre la Triplice.

En ce même mois de janvier 1913, un intéressant rapport du capitaine des carabiniers de Rhodes éclaire un peu plus la manière avec laquelle les autorités italiennes considèrent l'agitation nationaliste ${ }^{29}$. Les Anciens sont appelés “ noyau des intellectuels séditieux ”. Le capitaine des carabiniers raconte comment, en liaison avec le comité nationaliste d'Athènes, ils ont organisé une manifestation panhellénique lors d'une célébration religieuse présidée par le Métropolite Beniamino. Le rapport reprend la veine rhétorique inaugurée quelques jours auparavant, qui consiste à relativiser l'importance de la volonté nationale grecque en soulignant les vœux contraires des autres communautés :

“Même sans tenir compte des sentiments de la population turque et juive, qui constitue plus de la moitié des habitants de la ville de Rhodes, il est clair que la grande majorité des Rhodiens Grecs reste tranquille, étrangère aux menées politiques, et reconnaissante à notre Gouvernement ".

D'Ameglio avait fait avertir les démogérontes et le maire de l'interdiction de transformer la procession en manifestation, mais l'occasion était trop belle. La cérémonie de l'Epiphanie, en effet,

27. - Télégramme " rouge” au Secrétaire Général aux Affaires étrangères Di Martino, Rhodes, 15 janvier 1913. A.C.S., P.C.M., 1913, fasc. 1-2.

28. - Idem.

29. - Legione territoriale dei Carabinieri Reali di Napoli. Compagnia dell'Egeo, 20-1-1913. Circa una festa della religione greco ortodossa in Rodi, A.C.S., P.C.M., 1913, fasc. 1-2. 
constitue un moment privilégié de la vie civique citadine : les autorités urbaines (démogérontes, maire, conseil municipal) défilent dans les rues et descendent jusqu'à la mer pour une union symbolique.

Là encore, c'est le système urbain, et les références religieuses qui y sont liées, qui est mis au service de la revendication nationaliste. Pour les Grecs, de plus, parvenir à donner ce sens à la procession rhodienne, c'est prendre le dessus dans le jeu communautaire, et réduire l'expression de la politique urbaine à celle de la communauté grecque, ce qui est loin d'être facile en d'autres occasions, où les représentants des autres communautés se font entendre. Les carabiniers, lors de cette cérémonie, dispersent les "quelques fanatiques" sans ménagement. Le rapport précise qu'ils n'auraient pas pénétré dans les lieux de culte pour ce faire. A la fin de la manifestation, des Grecs s'étant rendus chez le consul de France, M. Lafon, pour demander protection, c'est devant le consulat que sont dispersés les derniers patriotes. Tous les enjeux de la vie insulaire sont réunis dans cette journée.

Quelques jours plus tard, d'Ameglio fait lui-même un rapport au Président du conseil ${ }^{30}$. Les agitateurs, qui auraient tenté de recommencer les manifestations le dimanche suivant, sont appelés "caporioni", chefs de quartier, autre indication intéressante du lien entre manifestation politique et institutions urbaines traditionnelles. Cette fois-ci d'Ameglio affirme n'avoir autorisé aucun débordement. Il s'inquiète cependant auprès de sa tutelle d'informations relatives à des livraisons clandestines d'armes.

De plus en plus, d'Ameglio prend des airs de gouverneur ottoman. Il s'adapte au cadre institutionnel local, et aux manières de réprimer les tentatives nationalistes. Il s'entend d'ailleurs fort bien avec le cadi de la ville, fonctionnaire ottoman resté en place.

Les protestations contre d'Ameglio affluent désormais de toutes parts. Les Grecs d'Alexandrie s'insurgent du fait que lors de la fête de l'Epiphanie les Italiens aient profané les églises (ce que nient les carabiniers). "Ce qui a été respecté par les Turcs est attaqué par les agents de l'Etat italien "31. Pour le général d'Ameglio cependant, à partir de février 1913, la situation est perçue comme stabilisée. Il est encore plus clair qu'avant que les îles ne seront ni rendues à l'Empire ni données à la Grèce. D'Ameglio en février reçoit aussi le soutien des communautés juive et musulmane ${ }^{32}$.

Les deux chefs de communauté se rendent à son bureau pour lui affirmer que face à l'impossibilité de voir l'île rendue à la domination ottomane, ils préfèrent qu'elle soit occupée de manière permanente par l'Italie ${ }^{33}$.

D'autres protestations grecques arrivent encore sur le bureau du Président du conseil, dont celle de la communauté de $\mathrm{Simi}^{34}$. Les communautés de la diaspora grecque s'organisent de plus en plus. Les télégrammes affluent, de même que les pétitions. C'est tout un mouvement politique qui se monte contre les Italiens. Pour l'Egypte, c'est un pharmacien, C. Yaloussis, qui orchestre la mobilisation. D'Amérique arrivent de nombreux télégrammes, alors que sur place à Rhodes les notables tentent de convaincre leurs compatriotes de ne pas s'expatrier. Les autorités religieuses lancent même plusieurs appels en ce sens, surtout à l'intention des habitants des petites îles et des villages ${ }^{35}$.

30. - Rapport du 29 janvier 1913, A.C.S., P.C.M., 1913, fasc. 1-2.

31. - Télégramme, janvier 1913, A.C.S., P.C.M., 1913, fasc. 1-2. Signé : Les Rhodiens en Egypte. Casulli, Casdagli, Drakides, Manolakis, Carayannis. Sur Alexandrie : Ilbert (Robert), Alexandrie, 1830-1930 : histoire d'une communauté citadine, Le Caire : Institut français d'archéologie orientale, 1996, 2 v., xxx, 886 p.

32. - A.C.S., P.C.M., 1913, fasc. 1-2. Voir en annexe.

33. - Rapport Ameglio, 5 février 1913, A.C.S., P.C.M., 1913, fasc. 1-2.

34. - Cette protestation est signée par les démogérontes et le maire. A.C.S., P.C.M., 1913, fasc. 1-2.

35. - Le Métropolite de Rhodes écrit ainsi à ses Popes et aux conseilllers municipaux en mai 1913 de donner des consignes pour éviter le départ des habitants grecs, qu'il qualifie de “trahison”. A.C.S., P.C.M., 1913, fasc. 1-2. 
Sur place, d'Ameglio a en revanche beaucoup de problèmes face à la résistance de la démogérontie urbaine et du maire de la ville. Autant au cours des mois précédents l'opposition était plutôt périphérique et rurale, autant à partir de la fin de l'année 1912, et pour toute l'année 1913, c'est Rhodes qui en est le centre. C'est d'abord Paolides, le maire de la ville, qui est destitué, à cause de réunions organisées conjointement par la mairie et la démogérontie communautaire ${ }^{36}$.

En mars, l'ex-maire de la ville fait publier par le Times de Londres un appel à l'union de Rhodes à la Grèce. San Giuliano, Ministre des Affaires étrangères, s'emploie de son mieux pour faire jouer ses réseaux londoniens et susciter des articles laudateurs sur l'action italienne face à la Turquie ${ }^{37}$. D'Ameglio expulse quant à lui Paolides ${ }^{38}$ qu'il avait déjà menacé à cause d'un télégramme envoyé au nouveau président de la République française quelques semaines plus tôt.

Celui qui était encore maire y écrivait, en union avec les démogérontes :

“ Présentant sincères félicitations peuple rhodien hellénique pour Votre nomination souhaitons carrière glorieuse pour le bien de la civilisation liberté des peuples. Démogérondie Rhôdes " ${ }^{39}$.

La presse française, à cette même période, dénonce aussi les méthodes d'Ameglio. Il est accusé d'avoir mis en place une police secrète et un bureau de la censure. A voir toutes les correspondances interceptées entre patriotes grecs que l'on trouve dans les archives, on ne peut que confirmer. Plusieurs notables de la ville tentent alors d'en appeler directement à Giolitti.

On trouve ainsi une lettre de Théodoridès :

“Ne permettez pas à un gouffre de s'ouvrir entre deux Nations sœurs créées pour s'aimer après tant de siècles de souffrances $" 40$.

Les institutions de la notabilité urbaine, dominées par les Grecs, sont particulièrement visées par la répression menée par d'Ameglio. Les réunions des démogéronties sont entravées, et le général fait tout son possible pour recruter des informateurs. Les notables municipaux de Rhodes font même appel à Ernesto Nathan, maire progressiste de Rome, pour lui demander d'intervenir auprès du gouvernement. Nathan ne répond pas, mais transmet ${ }^{41}$.

Durant le printemps et l'été 1913, d'Ameglio expulse encore de nombreux Grecs. Mais il est limogé, et remplacé par le général Marchi, qui prend ses fonctions en novembre ${ }^{42}$. Ameglio a échoué à se concilier les faveurs des institutions locales, et, entré en totale opposition, n'a pas su empêcher un fort sentiment anti-italien de se développer parmi la population grecque. Il n'a pas su non plus vivifier les institutions municipales ottomanes, où toutes les communautés sont représentées et qui auraient pu être un contrepoids. Il a laissé les institutions communautaires de la notabilité grecque prendre le dessus.

Dès lors la mairie n'était plus qu'une annexe du Conseil des Anciens grec. Il n'a pas su non plus user de diplomatie et de rhétorique pour se concilier une part de cette notabilité. Ou plutôt, la rhétorique initiale dont il a usé lui ayant été reprochée, il a ensuite appliqué jusqu'à la caricature les instructions venues de Rome.

Une des premières affaires dont doit traiter Marchi, successeur d'Ameglio au poste de gouverneur militaire, est encore une pétition, à l'occasion d'une visite à Rhodes du Duc des Abruzes, prince de la famille royale italienne. En cette occasion, en novembre 1913, la démogérontie et les autorités religieuses grecques orthodoxes s'unissent pour encore demander le rattachement à la Grèce ${ }^{43}$.

36. - Voir en annexe le décret. A.C.S., P.C.M., 1913, fasc. 1-2. Le texte est rédigé en trois langues : italien, grec et turc.

37. - A.C.S., P.C.M., 1913, fasc. 1-2.

38. - Rapport d'Ameglio au Président du conseil, 6 mars 1913. A.C.S., P.C.M., 1913, fasc. 1-2.

41. - A.C.S., P.C.M., 1913, fasc. 1-2., mars 1913.

42. - A.C.S., P.C.M., 1913, fasc. 1-2. 
La logique reste la même, et l'Italie, qui n'a d'autre choix que de rester, du fait de la situation internationale et de ses prétentions en Méditerranée, confirme n'avoir pas su amadouer la notabilité locale, et jouer des mécanismes de la société ottomane pour s'affirmer.

La conduite d'Ameglio s'apparentait pourtant dans l'île en bien des points à celle de ses prédécesseurs ottomans. Les autres communautés confessionnelles, juive et musulmane, s'étaient pourtant également manifestées. Dotées elles aussi d'organes de représentation, elle auraient pu être utilisées par le général d'Ameglio comme facteur d'équilibre. Quitte à jouer les Ottomans, autant aurait-il fallu le faire avec habilité.

A la fin de l'année 1912, par exemple, on trouve dans les archives de la Présidence du Conseil des Ministres, deux lettres émanant des deux communautés confessionnelles minoritaires. L'une, le 6 décembre, est adressée par le Tribunal religieux du Vilayet des Iles, avec siège à Rhodes, au Président de la Chambre des Députés italienne. Il s'agit d'une protestation contre un discours du député socialiste Chiesa qui, à la Chambre, a dénoncé la manière dont d'Ameglio se comportait en " gendarme pour le compte des Ottomans" en réprimant les aspirations nationales grecques. La lettre est signée du cadi de Rhodes, Ahmed Sciakia, du Mufti, Sanis, et des " notables " et Anciens de la communauté musulmane, Mehmed Sadik, Hasan Basri et Mehmed Kemal.

Ils y soulignent combien la communauté musulmane est satisfaite de l'action du général, et combien Rhodes ne se résume en rien à la communauté grecque. Ils y déclarent que le gouvernement ottoman n'a jamais non plus persécuté les Grecs. "La liberté et les privilèges donnés aux habitants des Sporades sous le gouvernement ottoman sont de plus confirmés par les firmans $q u ' i l s$ ont reçus ${ }^{~}{ }^{44}$. La lettre, qui a peut-être été suscitée par d'Ameglio, montre au moins que cette stratégie, qu'il n'a ensuite guère utilisée, était une voie possible face à la radicalisation de la césure confessionnelle, qu'il a finalement favorisée.

L'autre document émane de la communauté juive. C'est une lettre, en français, adressée au Grand Rabin d'Italie ${ }^{45}$. Là encore, il s'agit d'une louange de l'action d'Ameglio, destinée à contredire les attaques que subit le général dans la presse. Même si la manœuvre, là encore, est sans doute dirigée par le militaire, elle est au moins la preuve que les plus hautes institutions communautaires juives lui sont favorables.

Dans la ville de Rhodes, donc, sur trois communautés représentées dans le système ottoman par un conseil des notables, deux sont favorables à d'Ameglio, ce qui représente environ la moitié de la population.

Il est donc clair que le général, en 1913, n'a pas su jouer cette carte, en bon " ottoman " qu'il aurait dû être, puisque administrant un système hérité auquel il n'avait rien changé.

Il aurait pu aussi, plutôt que de permettre une symbiose entre institutions communautaires grecques et municipalité, ce qui l'a poussé à se mettre à dos les notables grecs définitivement, favoriser un fonctionnement de type ottoman des institutions municipales.

Faute de l'avoir compris, la posture répressive a été pour lui la seule issue avant une destitution inéluctable.

43. - A.C.S., P.C.M., 1913, fasc. 1-2. Voir le texte en annexe.

44. - A.C.S., P.C.M., 1913, fasc. 1-2.

45. - A.C.S., P.C.M., 1913, fasc. 1-2. La lettre est signée par Michael Samuel Menache, Président du conseil communal (communautaire) juif. 